\title{
Preface
}

\section{Prevention, Screening, and Treatments for Obstructive Sleep Apnea: Beyond Positive Airway Pressure (PAP)}

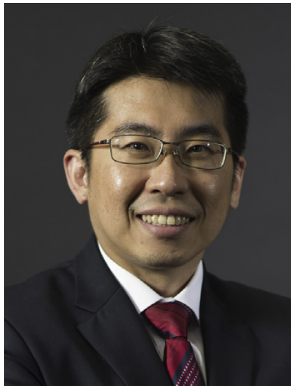

Song Tar Toh, MBBS, MRCSEd, MMED (ORL), MMED (Sleep Med), FAMS (ORL)

Editor
The description of obstructive sleep apnea (OSA) syndrome in the 1970s was followed by the first wave of academic research to understand the pathophysiology of sleep-related abnormal breathing and upper airway obstruction, to determine its extent in the general population, and its many dire medical consequences and associations. This was followed quickly by the description of positive airway pressure (PAP) in 1981 to treat it and a second wave of academic research describing various single-modality treatments, including surgical modifications of the upper airway, oral appliances, weight management, and myofunctional therapy.

There is no doubt that PAP is currently still the mainstay of treatment. However, further understanding of this prevalent heterogenous condition brought us to the third wave, whereby academic research data provided valuable insights to better manage this condition holistically. Different health care specialties joined this endeavor to meet the demands of this condition, whereby different therapeutic modalities are used and strategies deployed to potentially delay the progression and the development of medical consequences. It has brought to our attention the need for multidisciplinary collaborations.

As these three overlapping waves continue to build upon previous data and findings, we have to rethink the need for screening for this condition in high-risk groups and the pediatric population. We need to think of screening for potential asymptomatic medical conditions associated with OSA with the aim of preventing death by treating some of these serious medical conditions associated with it.

The complex relationships between genetic predisposition and susceptibility, environmental influence, obesity, nasal respiration, craniofacial growth, intrapharyngeal airway structures, control of respiration and sleep provide fresh perspectives on how this condition can be prevented and managed.

This issue of Sleep Medicine Clinics dedicated to "Screening, Prevention, and Treatment of OSA: Beyond PAP" looks at some of the cutting-edge thinking behind screening and prevention strategies and increasing population awareness and utility of different non-PAP treatment for us to win the fight against this condition and its chronic medical and neurocognitive consequences.

Song Tar Toh, MBBS, MRCSEd, MMED (ORL), MMED (Sleep Med), FAMS (ORL)

Department of Otolaryngology

Singapore General Hospital Singhealth Duke-NUS Sleep Centre National University of Singapore

Yong Loo Lin School of Medicine \& Duke-NUS Medical School

20 College Road, Level 5 Singapore 169856, Singapore

E-mail address: songtar@gmail.com 